\title{
Subcellular Locations of MOD5 Proteins: Mapping of Sequences Sufficient for Targeting to Mitochondria and Demonstration that Mitochondrial and Nuclear Isoforms Commingle in the Cytosol
}

\author{
MAGDALENA BOGUTA, ${ }^{1} \dagger$ LESLIE A. HUNTER,,${ }^{1,2}$ WU-CHENG SHEN,${ }^{1}$ EDWIN C. GILLMAN,${ }^{1}$ \\ NANCY C. MARTIN ${ }^{3}$ AND ANITA K. HOPPER ${ }^{1,2 *}$ \\ Department of Biological Chemistry ${ }^{1}$ and Program in Cell and Molecular Biology, ${ }^{2}$ The Milton S. Hershey Medical \\ Center, The Pennsylvania State University, Hershey, Pennsylvania 17033, and Department of Biochemistry, University \\ of Louisville Medical School, Louisville, Kentucky $40292^{3}$
}

Received 14 September 1993/Returned for modification 6 November 1993/Accepted 4 January 1994

\begin{abstract}
MOD5, a gene responsible for the modification of $A_{37}$ to isopentenyl $A_{37}$ of both cytosolic and mitochondrial tRNAs, encodes two isozymes. Initiation of translation at the first AUG of the MOD5 open reading frame generates $\Delta^{2}$-isopentenyl pyrophosphate:tRNA isopentanyl transferase I (IPPT-I), which is located predominantly, but not exclusively, in the mitochondria. Initiation of translation at a second AUG generates IPPT-II, which modifies cytoplasmic tRNA. IPPT-II is unable to target to mitochondria. The $\mathbf{N}$-terminal sequence present in IPPT-I and absent in IPPT-II is therefore necessary for mitochondrial targeting. In these studies, we fused MOD5 sequences encoding $\mathrm{N}$-terminal regions to genes encoding passenger proteins, pseudomature COXIV and dihydrofolate reductase, and studied the ability of these chimeric proteins to be imported into mitochondria both in vivo and in vitro. We found that the sequences necessary for mitochondrial import, amino acids 1 to 11, are not sufficient for efficient mitochondrial targeting and that at least some of the amino acids shared by IPPT-I and IPPT-II comprise part of the mitochondrial targeting information. We used indirect immunofluorescence and cell fractionation to locate the MOD5 isozymes in yeast. IPPT-I was found in two subcellular compartments: mitochondria and the cytosol. We also found that IPPT-II had two subcellular locations: nuclei and the cytosol. The nuclear location of this protein is surprising because the $A_{37} \rightarrow$ isopentenyl $A_{37}$ modification had been predicted to occur in the cytoplasm. MOD5 is one of the first genes reported to encode isozymes found in three subcellular compartments.
\end{abstract}

The Saccharomyces cerevisiae MOD5 gene encodes an enzyme that catalyzes the modification of tRNA at $A_{37}$, one base $3^{\prime}$ of the anticodon, to generate isopentenyl adenosine $\left(i^{6} \mathrm{~A}\right)$. This enzyme, $\Delta^{2}$-isopentenyl pyrophosphate:tRNA isopentenyl transferase (IPPT), is responsible for the modification of both mitochondrial and cytoplasmic tRNAs $(8,33)$ and is found in mitochondria as well as mitochondrion-depleted cell extracts (18). Thus, the MOD5 gene encodes proteins found in more than one subcellular compartment.

There are now many examples of other eukaryotic genes that code for proteins found in more than one subcellular location (reviewed in reference 34; see references cited in reference 18). Members of this family are found in higher eukaryotes as well as fungi and encode a wide variety of activities in addition to those involved in tRNA biosynthesis. We are interested in elucidating the mechanisms that are responsible for directing such gene products to their correct cellular compartments.

Our previous studies showed that MOD5 encodes two proteins which differ at their amino termini $(18,35,44)$. They are produced by initiation of translation at two alternative in-frame AUGs located at codons 1 and 12, respectively (18). Initiation at the first AUG generates an $\mathrm{N}$-terminally extended isozyme called IPPT-I, and initiation at the second AUG \footnotetext{
7072.

* Corresponding author. Phone: (717) 531-6008. Fax: (717) 531-

$\dagger$ Present address: Institute of Biochemistry and Biophysics, Polish Academy of Science, 02532 Warsaw, Poland.
}

generates an isozyme, IPPT-II, lacking the first 11 amino acids (aa) of the open reading frame (ORF). Alternative translation initiation at these AUGs is dictated, at least in part, by a translational readthrough of the first AUG of the ORF (44).

The results of studies employing in vitro mutagenesis of codons 1 and 12 of the MOD5 gene showed that a substantial portion of IPPT-I is found in mitochondria and is responsible for mitochondrial tRNA modification. IPPT-II, in contrast, does not localize to mitochondria and plays no role in mitochondrial tRNA modification in vivo (18). These studies showed that the first 11 aa unique to IPPT-I are necessary for mitochondrial targeting. However, they did not address whether the 11 aa comprise the entire targeting signal or whether peptide sequences shared by IPPT-I and IPPT-II play a role in mitochondrial targeting. One purpose of the studies described here was to define the regions of IPPT sufficient for mitochondrial targeting.

Another goal of our research was to determine the intracellular locations of the IPPT isozymes in yeast cells. Previously, assaying tRNA modification and subcellular fractionation of cell extracts, we found that not all of IPPT-I was delivered to mitochondria and that a functional pool of this activity was able to modify cytosolic tRNAs (18). Thus, IPPT-I appeared to be in at least two subcellular compartments. Our current studies using indirect immunofluorescence show that IPPT-I is found in mitochondria and the cytosol.

We expected to find IPPT-II in the cytosol. Although the modification of many nucleosides of tRNA occurs in the nucleus concurrent with other pre-tRNA processing steps (36), 


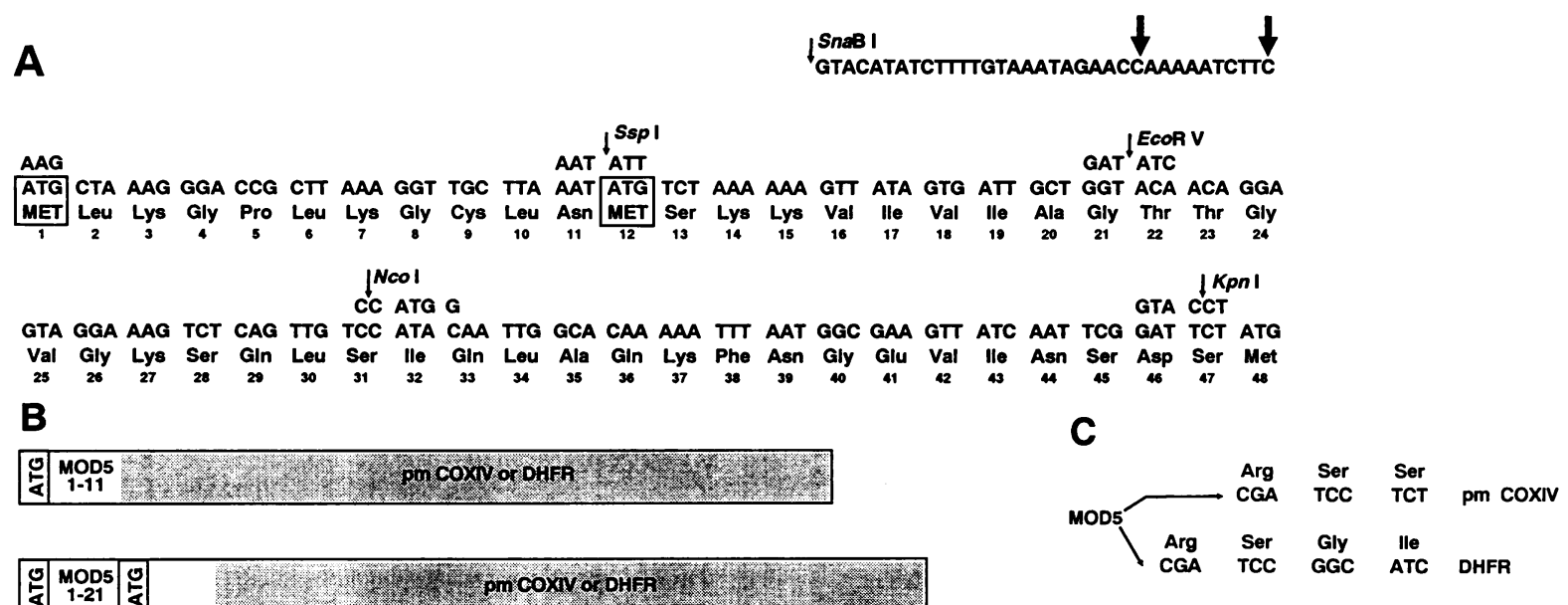

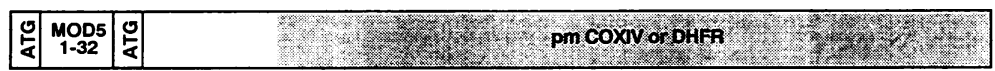

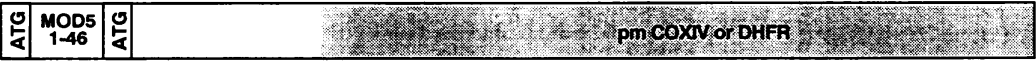

FIG. 1. Construction of hybrid genes. (A) Nucleotide sequence of $5^{\prime}$ end of the MOD5 gene. All inserts containing MOD5 start from the SnaB1 restriction site located 5' of the mRNA start sites (large arrows). The first and second AUG codons of the ORF are boxed. The new DNA sequences of each of the mutants and the resulting introduced restriction sites are given above the original sequence. (B) Schematic representation of the hybrid genes. Different-length MOD5 N-terminal coding regions (open box) were fused in frame to the region encoding aa 20 to 155 of COXIV or the entire DHFR-coding region (shaded boxes). (C) DNA sequences of fusion junctions.

three lines of evidence led to the prediction that the modification of $\mathrm{A}_{37}$ to $\mathrm{i}^{6} \mathrm{~A}_{37}$ in yeast cells would be catalyzed in the cytosol. First, in yeast cells, the rnal-1 mutation prevents intron excision and causes the accumulation of intron-containing pre-tRNAs (20) which do not contain $i^{6} \mathrm{~A}$ even though other modifications are present (27). However, it is not clear that an intron-containing pre-tRNA could serve as a substrate for $i^{6} A$ transferase. Second, when yeast pre-tRNA ${ }^{\text {Tyr }}$ was processed in Xenopus oocytes, $\mathrm{i}^{6} \mathrm{~A}$ modification followed intron removal and could be detected only on mature cytosolic tRNAs (36). Finally when mature tRNAs lacking $i^{6} \mathrm{~A}$ are injected into Xenopus oocytes, they can acquire this modification in the cytosol (19). These findings indicated that the order of the late events of tRNA processing is intron removal followed by tRNA export to the cytosol and by modification of A to $\mathrm{i}^{6} \mathrm{~A}$. We were therefore surprised to discover that IPPT-II is located in the nucleus as well as in the cytosol.

\section{MATERIALS AND METHODS}

Strains, media, and growth conditions. $S$. cerevisiae strains used were MD14A (MAT $\alpha$ SUP can1-100 ade2-1 his5-2 leu2-3,112 lys1-1 lys2-1 trp1 ura3-1 mod5-1 [18]), MT-8 (derivative of MD14A containing the mod5::TRP1 disruption [18]), WD1 (MAT $\alpha$ leu2 his3 ura3 coxIV::LEU2 [13]), D273-10B (47), and BJ2168 (MATa leu2 trp1 ura3-52 prb1-1122 pep4-3 prc1-407 gal2 [25]).

Yeast strains were maintained on YEPD medium or synthetic medium (22) lacking the appropriate nutritional ingredient. To determine growth rate, cells were inoculated into liquid synthetic medium lacking uracil and containing $3 \%$ (wt/wt) glycerol and $2 \%$ (wt/wt) lactic acid as carbon sources. To prepare cell fractions, yeast cells were grown on YEPgalactose (1\% yeast extract, $2 \%$ peptone, $2 \%$ galactose) medium supplemented with $100 \mu \mathrm{g}$ of adenine and uracil per ml. To prepare mitochondria to be used for in vitro import studies, cells were grown on synthetic medium containing lactate as the carbon source.

The Escherichia coli strains used were RR1 ( $\mathrm{F}^{-}$pro leu thi lacY Str ${ }^{\mathrm{r}} \mathrm{r}_{\mathrm{K}}{ }^{-} \mathrm{m}_{\mathrm{K}}{ }^{-}$hsdR hsdM endoI) and MC1066 (leuB trpC pyrF::Tn $5 \mathrm{r}_{\mathrm{K}}{ }^{-} \mathrm{m}_{\mathrm{K}}{ }^{-}$araT lacXZY rpsL). Yeast strains were transformed by using the alkali metal protocol of Ito et al. (24).

Plasmids and plasmid constructions. Plasmids containing wild-type $(M O D 5)$ or mutant alleles with alterations of the first (mod5-M1), second (mod5-M2), or first and second (mod5-M1, M2) ATGs inserted in low-copy-number (YCf50) or multicopy (YEp24 and pJDB207) vectors have been described previously (18). pMC4 encodes the precursor form of cytochrome oxidase subunit IV (COXIV) regulated by the yeast $A D H 1$ promoter (23). Plasmid pMSS encodes a pseudomature form of COXIV (pm COXIV) lacking the first 19 amino acids and is also regulated by the $A D H 1$ promoter (23). YCpADH1-mod5-M2COXIV and YCpADH1-mod5-M1-COXIV, which code for fusion proteins containing MOD5 aa 1 to 156 and 12 to 156 , respectively, fused to COXIV have been described previously (18).

Hybrid genes were constructed by a multistep procedure. Oligonucleotide mutagenesis (29) was used to introduce restriction sites at codons 11 and 12, 21 and 22, 32 and 33, and 46 and 47 (Fig. 1A). The oligonucleotides used were 5'-GGTT GCTTAAATATTTCTAA-3', 5'-GTGATTGCTGATATCA CAGGAGTA-3', 5'-GTTGTCCATGGAATTGGCA-3', and 5'-TATCAATTCGGTACCTATGCAGGTA-3', respectively. The mutant DNAs were digested with restriction enzymes to generate fragments with one end at the SnaB1 site (Fig. 1A) upstream of the MOD5 gene and the other at the mutagenized codon. The fragments were placed in-frame into the coding sequences of pm COXIV or mouse dihydrofolate reductase (DHFR) (Fig. 1B). The vectors used to generate these fusions were G18, a centromere-containing yeast shuttle vector that possesses the coding region for mature COXIV preceded by a 
polylinker and the yeast $A D H 1$ promoter (46), and pDS512, a vector that replicates in $E$. coli and contains the mouse DHFR sequence preceded by a polylinker region and the $P_{N 25} \times 10$ promoter (45). The plasmids containing MOD5-COXIV fusion sequences are referred to as 1-11 MOD5-COXIV, 1-21 MOD5-COXIV, 1-32 MOD5-COXIV, and 1-46 MOD5COXIV and encode, respectively, MOD5 aa 1 to 11,1 to 21,1 to 32 , and 1 to 46 fused to COXIV. Those containing MOD5DHFR fusion sequences are referred to as 1-11 MOD5DHFR, 1-21 MOD5-DHFR, 1-32 MOD5-DHFR, and 1-46 MOD5-DHFR. Note that each fusion junction joins the MOD5 sequence to an arginine of the passenger protein (Fig. 1C). All constructions were verified by DNA sequencing.

For in vitro transcription-translation reactions, the fusion constructs described above were digested with SnaB1 and HindIII and were subcloned into the pGEM3 vector at the SmaI and HindIII sites downstream of the T7 promoter.

In vitro transcription-translation and import of proteins into mitochondria. Plasmids were purified (28), and the DNAs were linearized by digestion with HindIII. The DNAs were transcribed with $\mathrm{T} 7$ polymerase, and the mRNAs were capped as specified by the manufacturer (Promega Corp.). The capped RNAs were translated by using a rabbit reticulocyte system (Promega Corp.) in the presence of ${ }^{35} \mathrm{~S}$-labeled amino acids. Mitochondria were isolated by the procedure of Daum et al. (7). Protein concentration was estimated by a modification of the method of Peterson (37). Mitochondria were incubated with the radiolabeled proteins (17) in a total volume of $200 \mu \mathrm{l}$ containing $200 \mu \mathrm{g}$ of mitochondrial protein, $10 \mu \mathrm{l}$ of translation mixture, $40 \mathrm{mM} \mathrm{KCl}$, and, where indicated, $2 \mu \mathrm{g}$ of valinomycin per ml. The import reaction was carried out for 30 min at $30^{\circ} \mathrm{C}$. For digestion of externally located polypeptides, proteinase $\mathrm{K}$ was added to $250 \mu \mathrm{g} / \mathrm{ml}$, and the mixture was incubated for $15 \mathrm{~min}$ at $0^{\circ} \mathrm{C}$. The protease was inhibited with phenylmethylsulfonyl fluoride. Mitochondria were reisolated by centrifugation through a sucrose cushion (15), suspended in $10 \mu$ l of breaking buffer (7), and analyzed on a sodium dodecyl sulfate (SDS) $-12 \%$ polyacrylamide gel. For the protein standard, the equivalent of $2 \mu$ l of the entire translation mixture was applied to the acrylamide gel.

Indirect immunofluorescence. Indirect immunofluorescence experiments were carried out by modification of the procedures of Kilmartin and Adams (26) and Pringle et al. (40). The first variation involved diluting the poly-L-lysine stock solution (Sigma Chemical Co.) 1:10 in water, which results in a significant reduction of background fluorescence. Subsequently we have totally eliminated use of poly-L-lysine. Second, the fixation time with formaldehyde was reduced from $2 \mathrm{~h}$ to 20 or 30 min. The MOD5 antigen was detected by an affinity-purified MOD5-specific antibody (18) in a 1:5 dilution. A 1:150 dilution of a fluorescein isothiocyanate-conjugated goat anti-rabbit antibody (Jackson Laboratory) was used as the secondary antibody. Cells were viewed with a Nikon Microphoto fluorescence microscope and a Nikon Plan Apo $60 \times$ objective.

Cell fractionation and Western blot (immunoblot) analysis. Cell fractionation and subsequent protein blot analyses were performed as described by Shen et al. (42). To detect Mod5p, the blots were incubated overnight with MOD5 antibody at a 1:300 dilution. The secondary antibody was an alkaline phosphatase-conjugated goat anti-rabbit antibody (Jackson Immuno Research Laboratories, Inc.) used at a 1:7,500 dilution for 2 to $4 \mathrm{~h}$. Rna1p was detected by using anti-Rna1p serum 6142 (21) overnight at a dilution of 1:100,000. A horseradish peroxidase-linked donkey anti-rabbit antibody (1:7,500 2 to 4 h) was the secondary antibody. Antibody was detected by using an enhanced chemiluminescence kit (Amersham). To detect
TABLE 1. In vivo complementation of coxIV::LEU2 disruption by $M O D 5-C O X I V$ fusion genes ${ }^{a}$

\begin{tabular}{lclc}
\hline Plasmid & $\begin{array}{c}\text { MOD5 } \\
\text { coding } \\
\text { sequence } \\
\text { (aa) }\end{array}$ & $\begin{array}{c}\text { COXIV cod- } \\
\text { ing sequence }\end{array}$ & $\begin{array}{c}\text { Generation } \\
\text { time (h) }\end{array}$ \\
\hline 1-11 MOD5-COXIV & $1-11$ & pm COXIV & 13 \\
1-21 MOD5-COXIV & $1-21$ & pm COXIV & 4.5 \\
1-32 MOD5-COXIV & $1-32$ & pm COXIV & 12.5 \\
1-46 MOD5-COXIV & $1-46$ & pm COXIV & 4.5 \\
YCpADH1-mod5- & $1-156$ & pm COXIV & 5.5 \\
M2-COXIV & $12-156$ & pm COXIV & No growth \\
YCpADH1-mod5- & & COXIV & 4.5 \\
M1-COXIV & & pm COXIV & No growth \\
pMC4 & & & \\
pMSS & & &
\end{tabular}

${ }^{a}$ Growth on glycerol-lactate liquid medium at $30^{\circ} \mathrm{C}$ was monitored by spectroscopy $\left(A_{6 \%}\right)$.

Nop1p, we used mouse monoclonal antibody A66 (a gift from A. Caplan, Chapel Hill, N.C.) at overnight 1:10,000 dilution and horseradish peroxidase-linked sheep anti-mouse antibody $(1: 7,500,2$ to $4 \mathrm{~h})$ followed by chemiluminescence detection.

\section{RESULTS}

The first 11 aa of IPPT-I are not sufficient for efficient mitochondrial import. A simple model that could account for the fact that IPPT-I but not IPPT-II localizes to mitochondria would be that the 11-aa N-terminal extension of IPPT-I provides the information that is necessary and sufficient for mitochondrial targeting. Our previous studies showed that these amino acids are necessary for protein import, but they did not address whether they are sufficient (18). Our strategy to determine which amino acids of IPPT-I provide mitochondrial targeting information was to examine the ability of $\mathrm{N}$-terminal regions to function as a surrogate targeting sequence for proteins unable to be imported into the mitochondria.

We used the system devised by Hurt et al. (23) to study mitochondrial import in vivo. Briefly, yeast cells require mitochondrial COXIV to grow on nonfermentable carbon sources. A strain with a disrupted chromosomal COXIV gene $(\operatorname{coxIV}:: L E U 2)$ and a plasmid encoding a form of COXIV (pm COXIV) lacking mitochondrial targeting sequences is unable to grow on media containing glycerol and lactate as carbon sources. The same strain will grow on these media if the plasmid contains an in-frame mitochondrial targeting sequence fused to the beginning of the pm COXIV ORF.

We previously showed that a gene containing codons 1 to 156 of MOD5 fused in-frame to pm COXIV and expressed under the control of the yeast $A D H 1$ promoter was able to complement the coxIV::LEU2 mutation, since a strain with this construct has nearly the same generation time $(\sim 5 \mathrm{~h})$ on media containing glycerol and lactate as the carbon sources as does a strain with a wild-type COXIV gene (18). In contrast, plasmids lacking the COXIV mitochondrial targeting sequence (pMSS) or lacking the first 11 codons of MOD5 (YCpADH1-mod5M1-COXIV) are unable to complement the coxIV::LEU2 allele (Table 1).

In these studies, we generated new hybrid genes that contain MOD5 codons 1 to 11,1 to 21,1 to 32 , and 1 to 46 fused with pm COXIV (Fig. 1). These fusions, designated 1-11 MOD5COXIV, 1-21 MOD5-COXIV, 1-32 MOD5-COXIV, and 1-46 MOD5-COXIV, were tested for the ability to complement coxIV::LEU2 (Table 1). A plasmid containing only the first 11 
aa of MOD5, 1-11 MOD5-COXIV, is unable to provide the information for full complementation of coxIV::LEU2, since strain WD1 with this plasmid grows very slowly, with a generation time of $13 \mathrm{~h}$. In contrast, a plasmid containing the first 21 or 46 codons of $M O D 5$ provides the information for complete complementation of coxIV::LEU2; WD1 cells containing plasmids 1-21 MOD5-COXIV and 1-46 MOD5COXIV grow with a generation time of $4.5 \mathrm{~h}$ and are indistinguishable from strains harboring a plasmid with the wild-type COXIV gene (pMC4) or a fusion of codons 1 to 156 of MOD5 fused to pm COXIV (YCpADH1-MOD5-COXIV).

A simple interpretation of these data would be that amino acids 1 to 21 provide information needed for efficient mitochondrial targeting. However, the phenotype of cells containing 1-32 MOD5-COXIV complicated this simple interpretation because WD1 cells containing this plasmid grow at the same slow rate as did cells harboring 1-11 MOD5-COXIV. Therefore, it also seemed feasible that both 1-11 MOD5COXIV and 1-32 MOD5-COXIV chimeric proteins possessed efficient mitochondrial targeting information, but that the hybrid fusion proteins had altered enzymatic activity.

To distinguish between these two interpretations, we studied the ability of the hybrid proteins to be imported into mitochondria in vitro. We also fused the same $\mathrm{N}$-terminal regions of MOD5 to a second passenger protein, DHFR. We were thereby able to investigate whether amino-terminal sequences derived from MOD5 direct passenger proteins into mitochondria in vitro with the same relative efficiencies as they do in vivo and whether import in vitro is independent of the passenger protein used.

Hybrid MOD5-COXIV and MOD5-DHFR sequences were transferred to the pGEM3 vector downstream of the T7 promoter, and these sequences were transcribed by $\mathrm{T} 7$ polymerase and translated in vitro, using a reticulocyte lysate system (see Materials and Methods). 1-11 MOD5-COXIV and 1-156 mod5-M2-COXIV generate a single protein in the reticulocyte lysate (lanes 1 in Fig. 2, IA and VA). Constructs containing MOD5 codons 1 to 21,1 to 32 , and 1 to 46 all possess an ATG at codon 12 that is the initiation of translation site of IPPT-II. Translation of the 1-21 and 1-32 constructs in the reticulocyte lysate generates two proteins, the faster migrating of which presumably arises from initiation at the internal AUG (Fig. 2, II and III, lanes 1). Translation of the 1-46 constructs presumably also generates two proteins, but these are not resolved (Fig. 2, IV, lanes 1) by polyacrylamide gel electrophoresis. The possibility that there are two proteins comigrating, one of which lacks mitochondrial targeting information, presents difficulties in assessing the efficiency of mitochondrial import (see below).

Radiolabeled proteins were tested for the ability to be imported into yeast mitochondria in vitro, using the procedure described by Daum et al. (7). For each construct, we assessed the ability of the fusion protein to associate with mitochondria in the presence and absence of a mitochondrial potential (Fig. 2; compare lanes 2 and 3) and determined whether proteins were imported into mitochondria by resistance to protease (Fig. 2, lanes 4) and sensitivity to the combination of valinomycin and protease (Fig. 2, lanes 5). For those constructs in which proteins initiating at the first and second AUGs are resolved, an estimate of the amount of protein imported into mitochondria can be assessed by comparing the quantity of protein generated in vitro (lanes 1 in Fig. 2 show $20 \%$ of the translation products) with the amount of protein that is protease resistant (Fig. 2, lanes 4).

In general, the in vitro import of DHFR chimeric proteins was more efficient than for the cognate COXIV chimeric

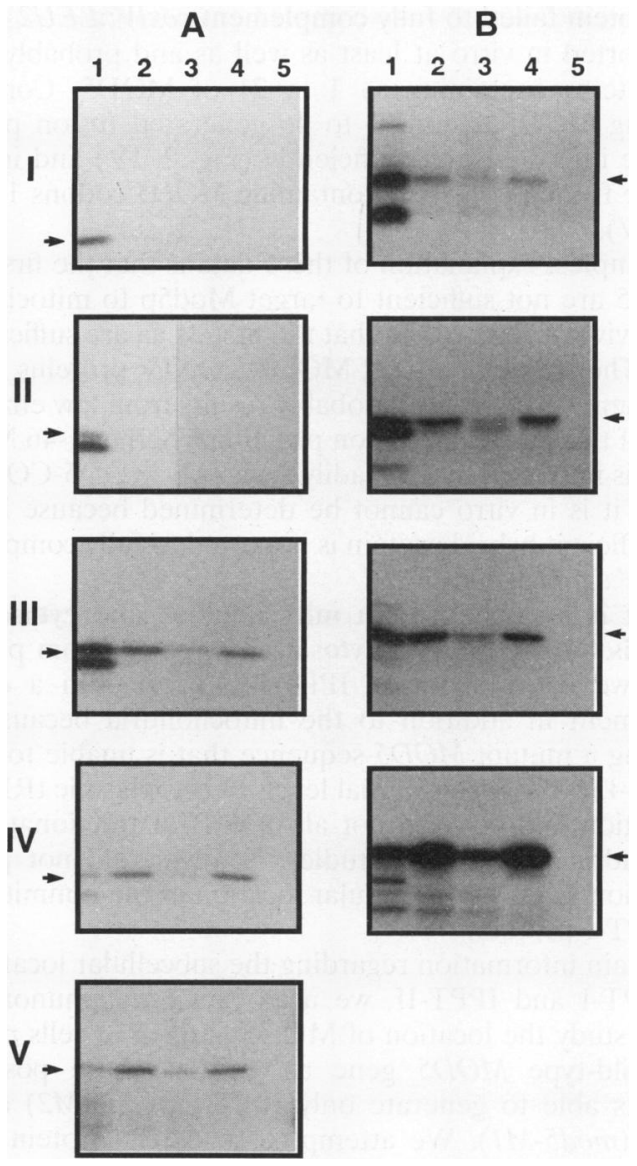

FIG. 2. In vitro import of MOD5 fusion proteins into yeast mitochondria. The MOD5-COXIV (A) and MOD5-DHFR (B) fusion proteins were synthesized in vitro in the presence of $\left[{ }^{35} \mathrm{~S}\right]$ methionine. Isolated yeast mitochondria were added to the translation mix, and the reaction mixture was incubated at $30^{\circ} \mathrm{C}$ for $30 \mathrm{~min}$. Mitochondria were enriched by centrifugation. The mitochondrial proteins were resolved on a $12 \%$ polyacrylamide-SDS gel. The dried gel was exposed to photographic film. Lanes 1 contain $2 \mu \mathrm{l}$ of the translation mix prior to the addition of mitochondria. Lanes 2 to 5 each contain the equivalent of $10 \mu \mathrm{l}$ of the translation mix and were treated as follows: lanes 2, no treatment (import assay); lanes 3, addition of valinomycin $(2 \mu \mathrm{g} / \mathrm{ml})$ to the reaction mixture (binding assay); lanes 4 , import assay followed by treatment with proteinase $\mathrm{K}(25 \mu \mathrm{g} / \mathrm{ml})$; lanes 5 , binding assay followed by treatment with proteinase K. Shown are results for fusions that possess MOD5 codons 1 to 11 (I), 1 to 21 (II), 1 to 32 (III), 1 to 46 (IV), and 1 to 156 (V). The arrow points to the MOD5 fusion protein that presumably initiates translation at the first AUG.

proteins (compare Fig. 2A and B). We did not detect import of proteins containing the first 11 aa of MOD5 fused to pm COXIV, and we detected a very low level $(<2 \%)$ of import when the same 11 aa were fused to DHFR (Fig. 2, IA and IB). Thus, the first 11 aa which are necessary for mitochondrial import do not supply information to allow full complementation of coxIV::LEU2 in vivo or to target efficiently either of two passenger proteins to mitochondria in vitro.

Proteins containing the first 21 aa of MOD5 showed passenger-specific mitochondrial import. 1-21 MOD5-COXIV hybrid proteins were not imported, (Fig. 2, IIA), whereas $\sim 30 \%$ of the 1-21 MOD5-DHFR hybrid proteins were imported (Fig. 2, IIB). Constructs containing MOD5 codons 1 to 32 generated fusion proteins were imported regardless of the passenger protein (Fig. 2, III). Although the 1-32 MOD5-COXIV chi- 
meric protein failed to fully complement coxIV::LEU2 in vivo, it is imported in vitro at least as well as and probably better than proteins containing aa 1 to 21 of MOD5. Constructs containing MOD5 codons 1 to 46 generated fusion proteins that were imported quite efficiently (Fig. 2, IV) and indistinguishable from a construct containing MOD5 codons 1 to 156 (Fig. 2, V).

The simplest explanation of these data is that the first 11 aa of MOD5 are not sufficient to target Mod5p to mitochondria either in vivo or in vitro but that the first 21 aa are sufficient for import. The inability of 1-32 MOD5-COXIV proteins to fully complement coxIV::LEU2 probably results from low enzymatic activity of this particular fusion protein. Whether 1-46 MOD5COXIV is imported more readily than 1-21 MOD5-COXIV in vivo like it is in vitro cannot be determined because in both cases, sufficient hybrid protein is imported to fully complement the coxIV::LEU2 mutation.

IPPT-I is located in both mitochondria and cytosol, and IPPT-II is located in both cytosol and nuclei. From previous studies, we concluded that IPPT-I is located in a cellular compartment in addition to the mitochondria because cells containing a mutant $M O D 5$ sequence that is unable to generate IPPT-II possess substantial levels of cytoplasmic tRNA $i^{6} \mathrm{~A}$ modification and because not all of IPPT-I fractionates with mitochondria (18). These studies, however, did not provide information as to the subcellular location of the nonmitochondrial IPPT-I protein.

To obtain information regarding the subcellular locations of both IPPT-I and IPPT-II, we used indirect immunofluorescence to study the location of MOD5 antigen in cells possessing a wild-type MOD5 gene as well as those possessing constructs able to generate only IPPT-I $(\bmod 5-M 2)$ or only IPPT-II $(\bmod 5-M 1)$. We attempted to detect protein generated in roughly endogenous levels by using a single-copy vector containing the MOD5 ORF regulated by the MOD5 promoter sequence. However, we were unable to detect MOD5 antigen with use of this vector, nor were we successful in visualizing Mod5p when it was overproduced $\sim 10$-fold from plasmid YEpMOD (not shown). We therefore used plasmids pJDBMOD5, pJDBmod5-M2, pJDBmod5-M1, and pJDBmod5M1,M2, which have wild-type or mutant MOD5 sequences inserted into vector pJDB207, which achieves high copy levels in yeast cells $(2,18)$.

Figure 3 contains representative photographs of the results of studies using the pJDB constructs. Figures $3 \mathrm{~A}, \mathrm{C}, \mathrm{E}$, and $\mathrm{G}$ display the fluorescein isothiocyanate detection of the MOD5 immunoreactive complex; Fig. B, D, F, and H show 4',6diamidino-2-phenylindole staining of mitochondrial and nuclear DNA. Cells containing the MOD5 gene with mutations at both the first and second ATGs (pJDBmod5-M1,M2) cannot generate either IPPT-I or IPPT-II. Expression of pJDBmod5M1,M2 (Fig. 3G and $\mathbf{H}$ ) resulted in an undetectable level of MOD5 antigen. In contrast, cells harboring a wild-type gene produce elevated levels of both IPPT-I and IPPT-II. For these cells (Fig. 3A and B), the MOD5 antigen was spread throughout the cells, obscuring the detection of individual organelles.

Cells possessing pJDBmod5-M2 showed, as expected, antigen located in mitochondria. In addition, some of the antigen was found throughout the cytosol (Fig. 3E and F). These results are consistent with our previous data and suggest that the nonmitochondrial IPPT-I is located throughout the cytosol. Thus, it appears that IPPT-I has two subcellular locations: mitochondria and cytosol.

The studies of the indirect immunofluorescence localization of IPPT-II generated an unexpected result. We found that cells possessing pJDBmod5-M1 had a very strong MOD5 signal in the nucleus as well as detectable levels of Mod5p in the cytosol (Fig. 3C and D). We were surprised to find Mod5p in nuclei because the modification of $A_{37}$ to $i^{6} A_{37}$ on tRNA had been reported to occur not in nuclei but rather in the cytoplasm (19, 27, 36).

To confirm the nuclear location of IPPT-II, we partitioned cell extracts into subcellular fractions (42). The location of Mod5p was monitored by protein blot analysis following electrophoretic separation of proteins in various subcellular fractions. We could readily detect Mod5p in total extracts, the organelle-depleted cytoplasmic fraction, and the nuclear-enriched fraction of cells harboring pJDBMOD5 (not shown). We could also detect Mod5p in total, cytosol, and nuclear fractions of yeast strain BJ2168 (25), which has the relevant genotype MOD5 prb1-1122 pep4-3 prc1-407. However, we found that there was some mitochondrial contamination in the nuclear fraction, and we could not eliminate the possibility that the Mod5p antigen in the nuclear fraction was from mitochondrial IPPT-I (not shown). We therefore used yeast strain MT-8 (relevant genotype, mod5::TRP1), which was transformed with a low-copy-number centromere-containing vector that contains the mod5-M1 allele (18). Thus, in this strain, only IPPT-II is generated, and it is roughly in endogenous levels. The quantity of Mod5p in MT-8 cells is smaller than the quantity in BJ2168 cells, probably because of proteolytic degradation of Mod5p during fractionation. Nevertheless, we could detect the antigen in total, cytosol, and nuclear fractions (Fig. 4A). The identity of the nuclear fraction was confirmed because Nop1p, a nucleolar protein (1), was found in total extracts and the nuclearenriched fraction but there was very little of this protein in the organelle-depleted cytosol fraction (Fig. 4B). We used antiRna1p to confirm that the nuclear fraction was relatively free of cytosol. Rna1p, a cytosolic protein (21), was found in total extracts and in the cytosol-enriched fraction, but it was barely detected in the nuclear-enriched fraction (Fig. 4C).

The immunofluorescence and cell fractionation studies show that IPPT-II, like IPPT-I, has two subcellular locations: nuclei and cytosol. Thus, while only IPPT-I appears to be targeted to mitochondria and only IPPT-II appears to be targeted to nuclei, the cytoplasm has significant contributions from both isozymes. The locations of the isozymes within yeast and the mapping of regions necessary for efficient mitochondrial targeting are summarized in Fig. 5.

\section{DISCUSSION}

Mitochondrial targeting of IPPT-I. Most genes that code for proteins destined to mitochondria encode an amino-terminal sequence that is necessary and sufficient for mitochondrial location (reviewed in references 38 and 39). The studies of TRM1 and HTS1, genes that also encode enzymes found in more than one compartment, indicate that this category of proteins might employ a variation on the mechanism of mitochondrial import. For both TRM1 and HTS1, the Nterminal extension of the mitochondrial protein is not sufficient to bring a surrogate protein into mitochondria $(6,13)$. For TRM1, endogenous levels of protein lacking the $\mathrm{N}$ terminal extension can be imported into mitochondria (12), but for HTS1, only overexpressed levels of the protein lacking the extension provide detectable levels of mitochondrially located protein (6). Thus, for both TRM1 and HTS1, mitochondrial targeting information resides in a portion of the protein that is shared by the two isozymes. The amino-terminal extensions somehow provide information that increases the efficiency of mitochondrial import. Our studies show that as for TRM1 and HTS1, the amino-terminal extension of the mito- 

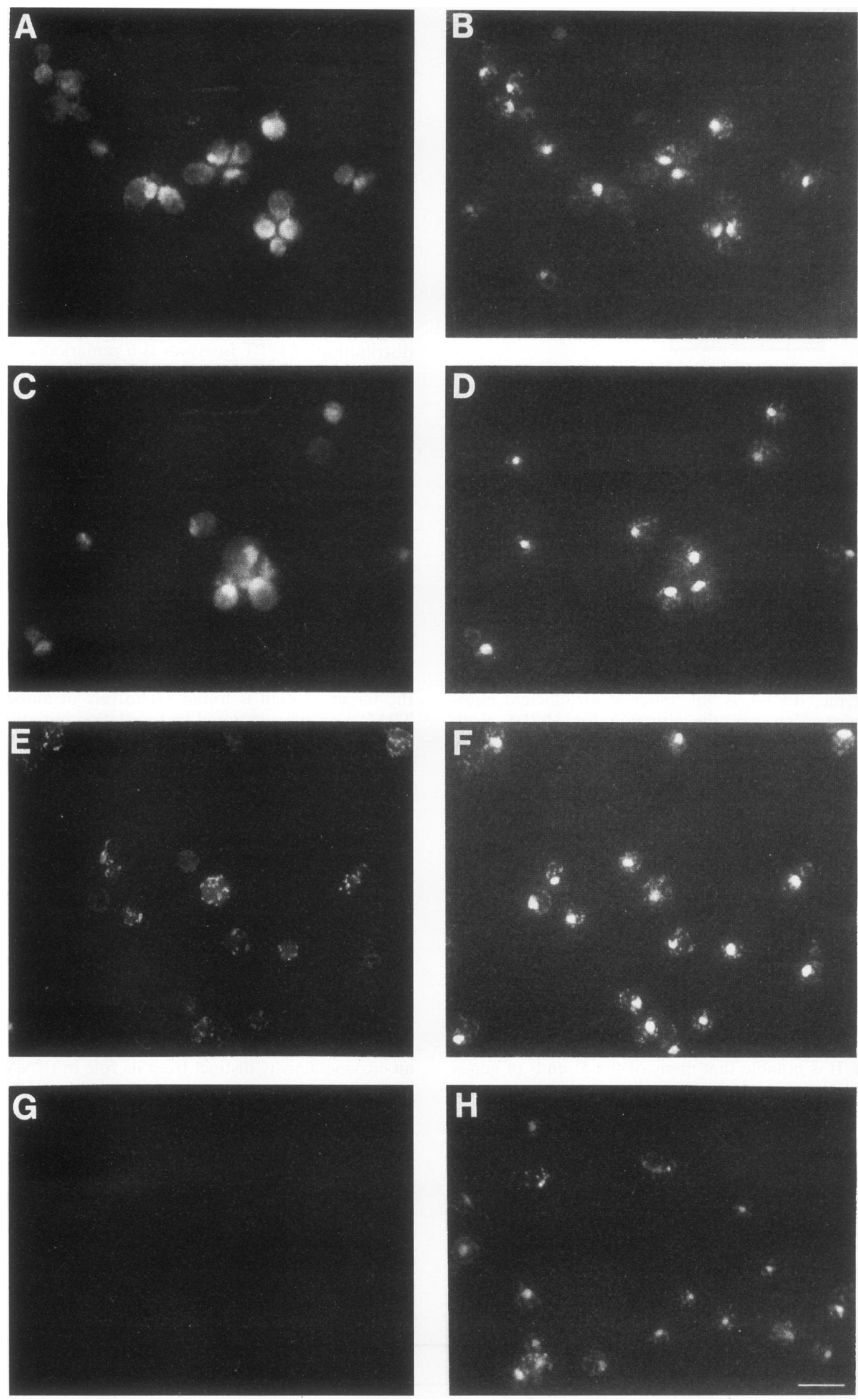

FIG. 3. Indirect immunofluorescence to determine the intracellular localization of IPPT isozymes. An affinity-purified anti-MOD5 antibody was used to detect the location of the MOD5 isozymes. (A, C, E, and G) Fluorescein isothiocyanate detection of MOD5 immunoreactive complexes; (B, D, F, and H) 4',6-diamidino-2-phenylindole (DAPI) staining of nuclear and mitochondrial DNA. (A and B) Yeast strain MT-8 harboring pJDBMOD5; (C and D) cells harboring pJDBmod5-M1; (E and F) cells harboring pJDBmod5-M2; (G and H) cells harboring pJDBmod5-M1,M2. 


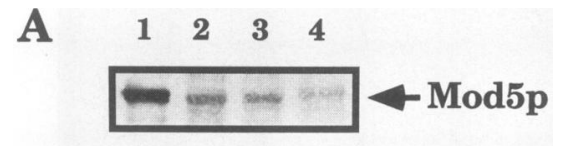

B

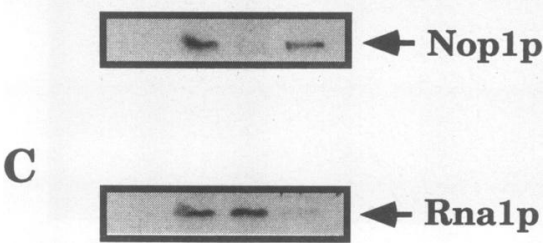

FIG. 4. Location of IPPT-II in subcellular fractions by immunodetection of proteins resolved on an SDS- $10 \%$ polyacrylamide gel. Lane 1 in each panel contains $<3 \mu \mathrm{g}$ of protein from MT-8 cells harboring the high-copy-number MOD5 plasmid, pJDBMOD5, prepared as described by Slusher et al. (44). Lanes 2 to 4 contain protein from MT-8 cells harboring a low-copy-number plasmid, YCfmod5-M1, encoding IPPT-II. Cell fractions were prepared as described by Shen et al. (42). Lanes 2 contain $40 \mu \mathrm{g}$ of total extracts; lanes 3 contain $40 \mu \mathrm{g}$ of organelle-depleted cytoplasmic enriched fractions; and lanes 4 contain $15 \mu \mathrm{g}$ of nuclear enriched fractions. (A) Detection of IPPT-II by chromogenic procedures; (B) detection of Nop1p by chemiluminescence; (C) detection of Rnalp by chemiluminescence. Arrows point to the particular proteins. Conditions for immunodetection are given in Materials and Methods.

chondrial isozyme, IPPT-I, is not sufficient for efficient mitochondrial import. However, the situation for MOD5 more closely resembles that for HTS1 than TRM1, because for both, $\mathrm{N}$-terminal peptides are necessary for mitochondrial import at least for endogenous levels of protein.

Of interest is the explanation for the functional cytoplasmic pool of IPPT-I. Although vectors that overexpress MOD5 were used for the immunofluorescence studies, it is unlikely that the cytosolic location of IPPT-I is an artifact of this overexpression because in endogenous levels, IPPT-I modifies cytoplasmic tRNA (18). Most genes encoding mitochondrial proteins do not have a cytoplasmic pool. In fact, there is a very close coupling of translation and mitochondrial import in yeast cells $(15,16)$. Mitochondrial targeting sequences are amphiphilic, rich in basic and hydroxylated residues, and deficient in acidic residues (reviewed in reference 48), and these peptides are predicted to have high hydrophobic moments in the range of 8 to $10 \mu \mathrm{H}(49)$. It is notable that many of the $5^{\prime}$ ends of genes encoding sorting enzymes depart from the consensus structure of mitochondrial targeting sequences $(3,4,13)$. In particular, calculation (11) of the hydrophobic moment of the first 18 amino acids of IPPT-I predict that this peptide has a $\mu \mathrm{H}$ of 5.27 , lower than those for most mitochondrial proteins (49). This lower moment is due to the presence of lysines instead of arginines in the amino terminus. Perhaps this departure from consensus renders the mitochondrial targeting sequence of IPPT-I inefficient, and therefore the presence of IPPT-I in the cytosol is a consequence of inefficient mitochondrial targeting. Alternatively, since tRNAs are predominant in the cytosol, it is possible that newly synthesized IPPT-I encounters these tRNAs and remains in the cytosol via association with tRNA. In any case, it appears that the cytosolic IPPT-I would not be one of those proteins reported to be imported cotranslationally into mitochondria.

Nuclear targeting of IPPT-II. We show that IPPT is localized to the cytoplasm, mitochondria, and nucleus. We are aware of only one other example of a gene, $C C A 1$, thought to encode similarly distributed enzymes (5). The subcellular distribution of IPPT isozymes is a remarkable result not only because MOD5 is one of the first genes demonstrated to encode isozymes sorted to three subcellular compartments but also because nonmitochondrial $i^{6} \mathrm{~A}$ modification was thought to occur in the cytoplasm. Interestingly, it appears that IPPTII, but not IPPT-I, is imported into the nucleus, even though IPPT-I must also contain the nuclear targeting information. It is conceivable that the extra N-terminal 11 aa of IPPT-I contribute to the formation of some secondary structure that masks the nuclear targeting sequence or in some other way prevents import. A similar phenomenon is suspected to operate for the yeast TRM1 gene, which encodes tRNA modification activities that are located in mitochondria and nuclei (14, 31). Cells producing the isozyme generated by translation of the entire TRM1 ORF (i.e., analogous to IPPT-I) have modified mitochondrial but not cytoplasmic tRNA, suggesting that the larger protein is found exclusively in mitochondria. The TRM1 isozyme generated by translation initiation at an internal AUG is located in nuclei and mitochondria. Thus, for both MOD5 and TRM1, the N-terminal extension appears to prevent nuclear import.

We have not identified the MOD5 nuclear targeting sequence yet. However, inspection of the DNA sequence provides clues as to its location. MOD5 lacks a stretch of consecutive basic amino acids that is necessary and sufficient to target proteins to nuclei in a wide variety of organisms, including yeasts (reviewed in reference 43). However, the MOD5 ORF does contain a sequence that matches the bipartite consensus nuclear targeting sequence $(9,41)$. This 17 -aa sequence is located at residues 408 to 424 of the C-terminal region of the MOD5 ORF (Fig. 5).

Of interest is the question of the biological function of the nuclearly localized IPPT-II. It is feasible that IPPT-II possesses an enzyme activity distinct from its role in tRNA modification and that activity resides in nuclei. Although seemingly far fetched, there are precedents for this. For example, mitochondrial tRNA synthetases of Neurospora crassa and $S$. cerevisiae function in both aminoacylation and pre-mRNA splicing (reviewed in reference 30). Another intriguing possibility is that the nuclear and cytosolic IPPT-II proteins modify distinct subsets of tRNAs. Previous studies of the location of modifi-

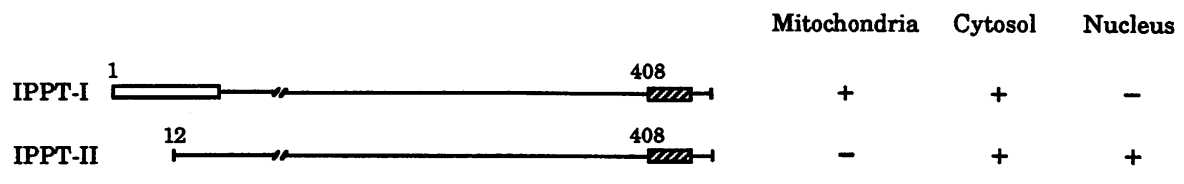

FIG. 5. Subcellular location of MOD5 isozymes. The lines depict the two forms of IPPT that differ at N termini. The open box indicates the region sufficient for efficient targeting to mitochondria. The hatched box indicates an amino acid sequence that bears similarity to bipartite nuclear localization signals. + , presence of the isozyme in a particular subcellular compartment; - , no isozyme was detected in the particular compartment. 
cation of $\mathrm{A}_{37}$ to $\mathrm{i}^{6} \mathrm{~A}_{37}$ assessed the modification of pre-tRNA encoded by intron-containing tRNA genes. We showed that IPPT-I that is localized in mitochondria and the cytosol is able to modify cytosolic tRNA ${ }^{\mathrm{Tyr}}(18)$. Therefore, it would appear that tRNA ${ }^{\mathrm{Tyr}}$, which is encoded by an intron-containing gene, can be modified in the cytosol, consistent with previous studies. Possibly those tRNAs encoded by genes lacking introns are modified in the nucleus. This proposal can be tested if we are able to generate alleles of MOD5 that produce IPPT-II that is located totally in the nucleus or the cytosol.

\section{ACKNOWLEDGMENTS}

This work was supported by an NIH GMS grant to N.C.M. and A.K.H.

We thank K. Verner for providing reagents and help with the studies involving mitochondrial in vitro import, $\mathrm{T}$. Zoladek for comments on the manuscript, and F. Cramer for manuscript preparation.

\section{REFERENCES}

1. Aris, J. P., and G. Blobel. 1988. Identification and characterization of a yeast nucleolar protein that is similar to a rat liver nucleolar protein. J. Cell Biol. 107:17-31.

2. Beggs, J. D. 1981. Multiplicopy yeast plasmid vectors, p. 383-390. In D. Von Wettstein, J. Friis, M. Kielland-Brandt, and A. Stenderup (ed.), Molecular genetics in yeast, vol. 16. Alfred Benzon Symposium. Munksgaard, Copenhagen.

3. Beltzer, J. P., S. R. Morris, and G. B. Kohlhaw. 1988. Yeast LEU4 encodes mitochondrial and nonmitochondrial forms of $\alpha$-isopropylmalate synthase. J. Biol. Chem. 263:368-374.

4. Chatton, B., P. Walter, J.-P. Ebel, F. Lacroute, and F. Fasiolo. 1988. The yeast VAS1 gene encodes both mitochondrial and cytoplasmic valyl-tRNA synthetases. J. Biol. Chem. 263:52-57.

5. Chen, J. Y., P. B. Joyce, C. L. Wolfe, M. C. Steffen, and N. C. Martin. 1992. Cytoplasmic and mitochondrial tRNA nucleotidyltransferase activities are derived from the same gene in the yeast Saccharomyces cerevisiae. J. Biol. Chem. 267:14879-14883.

6. Chiu, M. I., T. L. Mason, and G. R. Fink. 1992. HTS1 encodes both the cytoplasmic and mitochondrial histidyl-tRNA synthetase of Saccharomyces cerevisiae: mutations alter the specificity of compartmentation. Genetics 132:987-1001.

7. Daum, G., P. C. Bohni, and G. Schatz. 1982. Import of proteins into mitochondria: cytochrome b2 and cytochrome c peroxidase are located in the intermembrane space of yeast mitochondria. $\mathbf{J}$. Biol. Chem. 257:13028-13033.

8. Dihanich, M. E., D. Najarian, R. Clark, E. C. Gillman, N. C. Martin, and A. K. Hopper. 1987. Isolation and characterization of $M O D 5$, a gene required for isopentenylation of cytoplasmic and mitochondrial tRNAs of Saccharomyces cerevisiae. Mol. Cell. Biol. 7:177-184.

9. Dingwall, C., and R. A. Laskey. 1991. Nuclear targeting sequences-a consensus? Trends Biochem. Sci. 16:478-481.

10. Dowhan, W., C. R. Bibus, and G. Schatz. 1985. The cytoplasmically-made subunit IV is necessary for assembly of cytochrome c oxidase in yeast. EMBO J. 4:179-184.

11. Eisenberg, D., R. M. Weiss, and T. C. Terwillizer. 1982. The helical hydrophobic moment: a measure of the amphiphilicity of a helix. Nature (London) 229:371-374

12. Ellis, S. R., A. K. Hopper, and N. C. Martin. 1987. Amino-terminal extension generated from an upstream AUG codon is not required for mitochondrial import of yeast $N^{2}, N^{2}$-dimethylguanosinespecific tRNA methyltransferase. Proc. Natl. Acad. Sci. USA 84:5172-5176.

13. Ellis, S. R., A. K. Hopper, and N. C. Martin. 1989. Amino-terminal extension generated from an upstream AUG codon increases the efficiency of mitochondrial import of yeast $N^{2}, N^{2}$-dimethylguanosine-specific tRNA methyltransferases. Mol. Cell. Biol. 9:1611-1620

14. Ellis, S. R., M. J. Morales, J. M. Li, A. K. Hopper, and N. C. Martin. 1986. Isolation and characterization of the TRM1 locus, a gene essential for the $N^{2}, N^{2}$-dimethylguanosine modification of both mitochondrial and cytoplasmic tRNA in Saccharomyces cerevisiae. J. Biol. Chem. 261:9703-9709.

15. Fujiki, M., and K. Verner. 1991. Coupling of protein synthesis and mitochondrial import in a homologous yeast in vitro system. J. Biol. Chem. 266:6841-6847.

16. Fujiki, M., and K. Verner. 1993. Coupling of cytosolic protein synthesis and mitochondrial protein import in yeast. Evidence for cotranslational import in vivo. J. Biol. Chem. 268:1914-1920.

17. Gasser, S. M., G. Daum, and G. Schatz. 1982. Import of proteins into mitochondria: energy-dependent uptake of precursors by isolated mitochondria. J. Biol. Chem. 257:13034-13041.

18. Gillman, E. C., L. B. Slusher, N. C. Martin, and A. K. Hopper. 1991. MOD5 translation initiation sites determine $N^{t}$-isopentenylation modification of mitochondrial and cytoplasmic tRNA. Mol. Cell. Biol. 11:2382-2390.

19. Grosjean, H. Personal communication.

20. Hopper, A. K., F. Banks, and V. Evangelidis. 1978. A yeast mutant which accumulates precursor tRNAs. Cell 14:211-219.

21. Hopper, A. K., H. M. Traglia, and R. W. Dunst. 1990. The yeast $R N A 1$ gene product necessary for RNA processing is located in the cytosol and apparently excluded from the nucleus. J. Cell Biol. 111:309-321.

22. Hurt, D. J., S. S. Wang, Y.-H. Lin, and A. K. Hopper. 1987. Cloning and characterization of LOS1, a Saccharomyces cerevisiae gene that affects tRNA splicing. Mol. Cell. Biol. 7:1208-1216.

23. Hurt, E. C., U. Muller, and G. Schatz. 1985. The first twelve amino acids of a yeast mitochondrial outer membrane protein can direct a nuclear-coded cytochrome oxidase subunit to the mitochondrial inner membrane. EMBO J. 4:3509-3518.

24. Ito, H., Y. Fukuda, K. Murata, and A. Kimura. 1983. Transformation of intact yeast cells treated with alkali cations. J. Bacteriol. 153: $163-168$.

25. Jones, E. W. 1991. Tackling the protease problem in Saccharomyces. Methods Enzymol. 194:428-453.

26. Kilmartin, J. V., and A. E. Adams. 1984. Structural rearrangements of tubulin and actin during the cell cycle of the yeast Saccharomyces. J. Cell Biol. 98:922-933.

27. Knapp, G., R. C. Ogden, C. L. Peebles, and J. Abelson. 1979. Splicing of yeast tRNA precursors: structure of the reaction intermediates. Cell 18:37-45.

28. Krieg, P. A., and D. A. Melton. 1987. In vitro RNA synthesis with SP6 RNA polymerase. Methods Enzymol. 155:397-415.

29. Kunkel, T. A., J. D. Roberts, and R. A. Zakour. 1987. Rapid and efficient site-specific mutagenesis without phenotypic selection. Methods Enzymol. 154:367-382.

30. Lambowitz, A. M., and P. S. Perlman. 1990. Involvement of aminoacyl-tRNA synthetases and other proteins in group I and group II intron splicing. Trends Biochem. Sci. 15:440-444.

31. Li, J. M., A. K. Hopper, and N. C. Martin. 1989. N2,N2-dimethyl guanosine-specific tRNA methyltransferase contains both nuclear and mitochondrial targeting signals in Saccharomyces cerevisiae. J. Cell Biol. 109:1411-1419.

32. Maniatis, T., E. F. Fritsch, and J. Sambrook. 1982. Molecular cloning: a laboratory manual. Cold Spring Harbor Laboratory, Cold Spring Harbor, N.Y.

33. Martin, N. C., and A. K. Hopper. 1982. Isopentenylation of both cytoplasmic and mitochondrial tRNA is affected by a single nuclear mutation. J. Biol. Chem. 257:10562-10565.

34. Martin, N. C., and A. K. Hopper. Review in preparation.

35. Najarian, D., M. E. Dihanich, N. C. Martin, and A. K. Hopper. 1987. DNA sequence and transcript mapping of MOD5: features of the $5^{\prime}$ region which suggest two translational starts. Mol. Cell. Biol. 7:185-191.

36. Nishikura, K., and E. M. De Robertis. 1981. RNA processing in microinjected Xenopus oocytes: sequential addition of base modifications in the spliced transfer RNA. J. Mol. Biol. 145:405-420.

37. Peterson, G. L. 1977. A simplification of the protein assay method of Lowry et al. which is more generally applicable. Anal. Biochem. 83:346-356.

38. Pfanner, N., and W. Neupert. 1990. The mitochondrial protein import apparatus. Annu. Rev. Biochem. 59:331-353.

39. Pon, L., and G. Schatz. 1991. Biogenesis of yeast mitochondria, p. 333-406. In J. R. Broach, E. W. Jones, and J. R. Pringle (ed.), The molecular and cellular biology of the yeast Saccharomyces: ge- 
nome dynamics, protein synthesis and energetics. Cold Spring Harbor Laboratory Press, Cold Spring Harbor, N.Y.

40. Pringle, J. R., A. E. Adams, D. G. Drubin, and B. K. Haarer. 1991. Immunofluorescence methods for yeast. Methods Enzymol. 194: 565-602.

41. Robbins, J. S., M. Dilworth, R. A. Laskey, and C. Dingwall. 1991. Two independent basic domains in nucleoplasmin nuclear targeting sequence: identification of a class of bipartite nuclear targeting sequence. Cell 64:615-623.

42. Shen, W.-C., D. Selvakumar, D. R. Stanford, and A. K. Hopper. 1993. The Saccharomyces cerevisiae LOS1 gene involved in pretRNA splicing encodes a nuclear protein that behaves as a component of the nuclear matrix. J. Biol. Chem. 268:19436-19444.

43. Silver, P. A. 1991. How proteins enter the nucleus. Cell 64:489497.

44. Slusher, L. B., E. C. Gillman, N. C. Martin, and A. K. Hopper. mRNA leader length and initiation codon context determine alternative AUG selection for the yeast gene MOD5. Proc. Natl. Acad. Sci. USA 88:9789-9793.

45. Stueber, D., I. Ibrahimi, D. Cutler, B. Dobberstein, and H. Bujard. 1984. A novel in vitro transcription-translation system: accurate and efficient synthesis of single proteins from cloned DNA sequences. EMBO J. 3:3143-3148.

46. van Loon, A. P., and G. Schatz. 1987. Transport of proteins to the mitochondrial intermembrane space: the 'sorting' domain of the cytochrome $\mathrm{c} 1$ presequence is a stop-transfer sequence specific for the mitochondrial inner membrane. EMBO J. 6:2441-2448.

47. Verner, K., and B. D. Lemire. 1989. Tight folding of a passenger protein can interfere with the targeting function of a mitochondrial presequence. EMBO J. 8:1491-1495.

48. Verner, K., and G. Schatz. 1988. Protein translocation across membranes. Science 241:1307-1313.

49. von Heijne, G. 1986. Mitochondrial targeting sequences may form amphiphilic helices. EMBO J. 5:1335-1342. 